\title{
Great Lakes Toxic Chemical Decision Support System
}

\author{
(1)Booty, W.G., (1)Lam, D.C.L., (2)Tseng, T., (3)Smith, I. and (4)Siconolfi, P.
}

(1) National Water Research Institute, Environment Canada, 867 Lakeshore Rd., Burlington, Ontario, Canada L7R 4A6 Ph:(905)336-4718, Fax: (905)336-4972, email:bill.booty@cciw.ca

(2) Environmental Protection Branch, Environment Canada, 4905 Dufferin St., Downsview, Ontario, Canada M3H $5 T 4$ Ph: (416)739-5853, Fax: (416)739-4159, email:

tsengt@aestor.am.doe.ca@igw

(3) Ontario Ministry of Environment and Energy, 40 St. Clair Ave. W., Toronto, Ontario, Canada M4V 1M2 Ph: (416) 314-7996, Fax: (416) 314-3924, email:smithi@gov.on.ca

(4) ES Aquatic Inc., 376 Woolwich St., Guelph, Ontario, Canada N1H 3W7 Ph: (905) 336-6408, Fax: (905)336-4582, email: patrick@charon.cciw.ca

\begin{abstract}
In support of the 1994 Canada-Ontario Agreement (COA) to reduce pollutants in the Great Lakes, a decision support system is being developed and applied to answering management questions regarding the implementation and postauditing of the zero discharge and virtual elimination strategies for Tier I and Tier II toxic chemicals. The RAISON for Windows Decision support system, which was developed at the National Water Research Institute, Environment Canada, is the software that the application is based upon. It integrates data, text, maps, satellite images, pictures, video and other knowledge input. A library of software functions and tools are available for selective extraction of spatial and temporal data that can be analysed using spatial algorithms, models, statistics, expert systems, neural networks, and other information technologies. Results of the analyses can be shown as combinations of maps, graphs, contours, and animations of model outputs in 2 and 3 dimensions. Examples are presented of the various data analysis and visualization techniques being used within the Great Lakes for providing essential knowledge on the fate and effects of Tier I and Tier II toxic chemicals.
\end{abstract}

\section{Keywords}

Decision Support System; Simulation Application; User Interfaces

\section{INTRODUCTION}

The Canada-Ontario Agreement (COA) to reduce pollutants in the Great Lakes calls for the virtual elimination of thirteen Tier I and twenty-six Tier II chemicals which are known to be toxic, persistent, and bioaccumulative. Information on the emissions and background levels of the Tier I and Tier II chemicals are stored in various databases in federal, state, and provincial agencies. The data are collected and measured with varying levels of error and uncertainty. Many of these chemicals require sophisticated analytical chemistry methods to make measurements and may only be accurate to the "order of magnitude" level. The challenge is how to make the best of the measured data and the existing body of scientific knowledge for qualifying, if not quantifying, the fate and pathways of these substances in the context of designing, implementing and post auditing the zero discharge and virtual elimination strategies.

The first stage of the application of the decision support system is to collect all geo-referenced and non geo-referenced data on sources, releases and loadings and associated qualifying information and then to progressively synthesize them using remark codes, QA/QC ratings, confidence ranking, statistical analysis, and expert system techniques for proper use of data, treatment of below-detection data, and data gaps.

Environmental Software Systeins Vol. 2 R. Denzer, D.A. Swayne \& G. Schimak (Eds.)

(1) IFIP 1997 Published by Chapman \& Hall 
The next stage is the implementation of models for prediction of air transport and loadings of contaminants at continental, regional, and local scales. Also, mass balance models are required for predictions of concentrations and residence times of chemicals within the lakes and surrounding watersheds. The operation of these models will also include the use of uncertainty propagation techniques such as sensitivity analysis, first order error analysis and Monte Carlo simulation. Data required to operate the models such as system geometry, hydrometeorological forcing data, and basic physical and chemical properties of the system that govern transport and transformation processes must also be entered into the system.

This paper describes the development and application of the prototype of the Great Lakes Toxic Chemical Decision Support System.

\section{SYSTEM DESIGN and APPLICATIONS}

\subsection{RAISON for Windows Decision Support System}

The RAISON for Windows Decision Support System shell that this system is based upon is the most recent version of RAISON (Regional Analysis by Intelligent Systems ON microcomputers) which has evolved over the past decade at the National Water Research Institute of Environment Canada (Lam and Swayne, 1993, Lam et al., 1995). The system consists of the following modules: Database; Spreadsheet; GIS/Layer; Statistics; Expert System; Contouring; Spatial Visualization; and Graphs. Its development has been client driven and its design is devoted to providing generic software tools for fast prototyping and practical implementation of environmental decision support systems. The architecture is open in design. All of the above system modules are directly linked within the RAISON system with functions and tools including modelling interfaces, neural network, uncertainty analysis, fuzzy logic, animation, visualization and optimization procedures. For inter-connectivity, RAISON uses crosstalk features (Windows Clipboard, OLE, DDE), as well as special linkage interfaces for import and export to many of the popular commercial database, spreadsheet, graphics, and GIS software packages.

The RAISON for Windows database utilizes Microsoft Access $2.0\left(^{*} . \mathrm{mdb}\right.$ files) as the database standard. It is a relational database management system that utilizes a Structured Query Language (SQL). There is also a Photo Database feature that allows the user to store graphic images of sites. The database is associated with site data and is activated by polygon retrieval.

The Spreadsheet is similar to other commercial spreadsheets and is used for editing and manipulating data with basic algebraic functions and formulas. It has been designed to be fully integrated with all of the other RAISON modules.

Within the RAISON system, maps can be handled in vector and raster formats. It references geographical locations using either latitude-longitude or Universal Transverse Mercator (UTM) coordinate systems. Maps are saved as snapshots within the system. Snapshots can be created from map files of other commercial GIS systems such as ArcInfo or MapInfo, etc. A number of map projections are supported such as Geo (flat), Polar, Mollweide, Lambert Conformal Conic, CEASN (Cylindrical Equal Area Spherical Normal) and Vertical Perspective (Sphere). The system also supports the use of a layered database. Layers are collections of spatial points, lines and/or polygons that have some attribute. A grid layer feature is available for use with models that use a regular grid and which process information according to individual grid cells. Layers may be overlaid using intersection and other Boolean operations.

A special set of statistics for use in environmental applications are available within the RAISON statistics module. These include basic statistics( mean, mode, median, percentiles, etc.), hypothesis tests ( parametric, non-parametric, ANOVA and time series), normal and log-normal distribution analysis and other methods such as principal component analysis, clustering analysis, multivariate regression and other techniques. 
The RAISON expert system is a rule-based system with fuzzy logic. As such it may be used for data classification or prediction of data. It may also be used to advise on scientific processes and knowledge limitations (e.g. the choice of models or model coefficients), etc. Rules are entered directly into a spreadsheet format. The variables used in the rules can be described by attributes which can be qualitative, numerical, logical or a range of values. A generic inference engine is used to organise the rule base and to make conclusions or a course of action such as search the database for further information, display results on maps, or call another expert system (Lam et al., 1995, Booty et al. 1993).

The RAISON contour module allows the user to interpolate spatial data onto a grid using two methods: 1) Inverse distance and 2) Kriging (local and global variograms). The interpolated values can be graphically represented on a map in the form of lines or solid regions. The image can be saved as a bitmap and can be used as an image file in the layer module. There is also the option of creating a horizontal contour plot or a vertical cross-section graph. The Kriging method also produces an error estimate which can be displayed as error lines.

The spatial visualization module contains a number of tools unique to RAISON. They have been designed to effectively group data into classes and to allow the results to be viewed on a georeferenced snapshot. One tool is called the Starlist which is a matrix of scattergrams for selected parameters. Additional scatterplots that are available include QQ plots, bivariate ellipsoid, and regression lines. Thematic mapping is very useful in that it allows you to colour site locations according to classes and boxplots. The sites are classified using any criteria that you define.

The Graph module contains the standard types of graphs (e.g. line, bar, XY, Time-Series) that are available in most commercial spreadsheets. Box plot graphs are also available and are created in conjunction with the statistics module. Once the graphs have been created, they may also be resized and pasted on to maps.

\subsection{Great Lakes Toxic Chemical Decision Support System}

\section{Data Screening and Analysis}

In Figure 1 the conceptual design and component integration of the system is shown. Before the data can be used for decision support they need to be categorised, screened and analysed. The first step was to segregate the data into 3 main types: Emissions, Loadings, and Background. The background data include all of the physical and chemical data required as input to the models and for model calibration/confirmation.

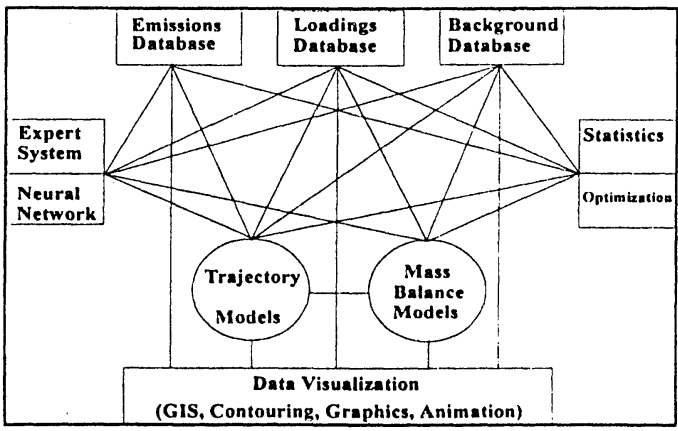

Figure 1 Great Lakes Toxic Chemical Decision Support System conceptual design. 
As is the case with all applications within RAISON, the Great Lakes Decision Support System operates as a separate project. In this case, as shown in Figure 2, it is called the Stream 2 Project. RAISON provides a starting procedure that creates the project structure so that data, metadata, and maps will be brought in, linked and made available for that project. There are different ways of accessing the data. Normally a user would only be working on one lake at a time. In that case, to quickly make all of the data and associated knowledge available, a spatial query is selected. This brings onto the screen a map of the Great Lakes, as shown in Figure 2. Each of the Great Lakes watersheds are represented by polygons. By moving the mouse pointer to one of the polygons and clicking on it, the selected polygon is highlighted, in this case Lake Ontario, as shown in Figure 2, and the system automatically links to the Lake Ontario database. At this point the user may select one of the data types for further examination. For example, as shown in Figure 3, Emissions have been selected and an Emissions Query window is opened. In this case Tier I chemicals have been selected and for both Canadian and US data. In the white box within the window those chemicals which are available in the database are automatically listed for selection. If lead (and its compounds) is selected, the location and size of the emissions can be shown on a map using the RAISON visualization feature "thematic mapping by class", as shown in Figure 3.

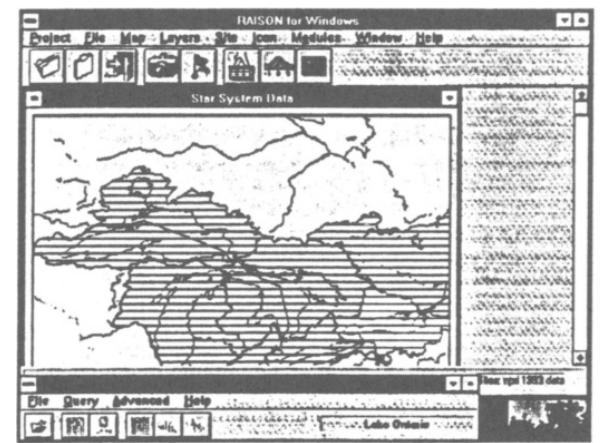

Figure 2 Great Lakes watershed showing Lake Ontario databases selected spatially by clicking on the Lake Ontario polygon.

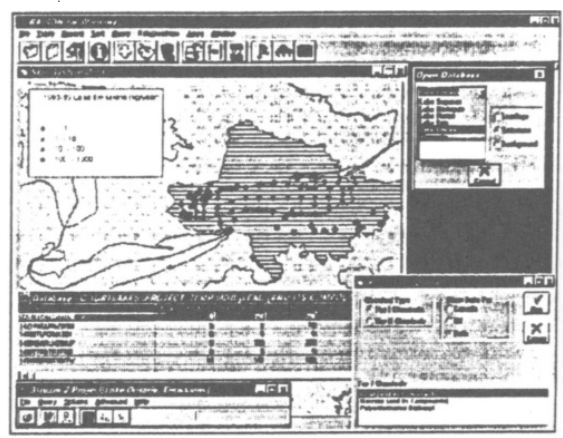

Figure 3 1992-93 lead emissions for Canadian and U.S. sources for the Lake Ontario watershed are shown by location and size in $\mathrm{Kg} /$ year using the RAISON thematic mapping by class visualization feature.

Statistical analyses may be quickly performed to further examine the characteristics of the data. For example, as shown in Figure 4, data for a number of chemicals have been brought directly into the statistics module from the database. Up to 12 different variables may be loaded at one time. In this case five chemicals measured as part of a Remedial Action Plan (RAP) study have been loaded and a more detailed analysis on the frequency distributions for lead is shown. In Figure 5 a multiple linear regression has been carried out with lead as the dependent variable and mercury and cadmium as the independent variables, showing the resulting multivariate equation and standard errors. 


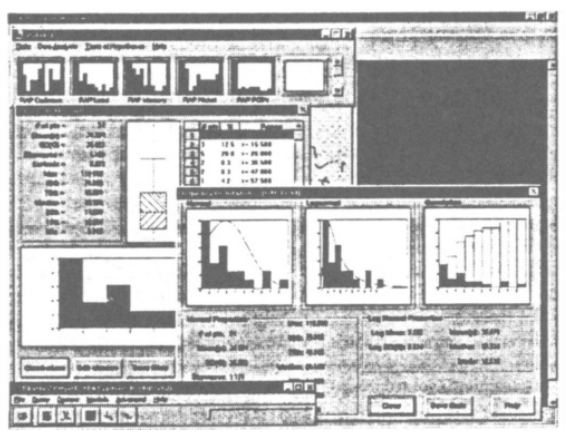

Figure 4 The frequency distributions of lead in sediments of Lake Ontario are easily calculated and graphed using the statistics module.

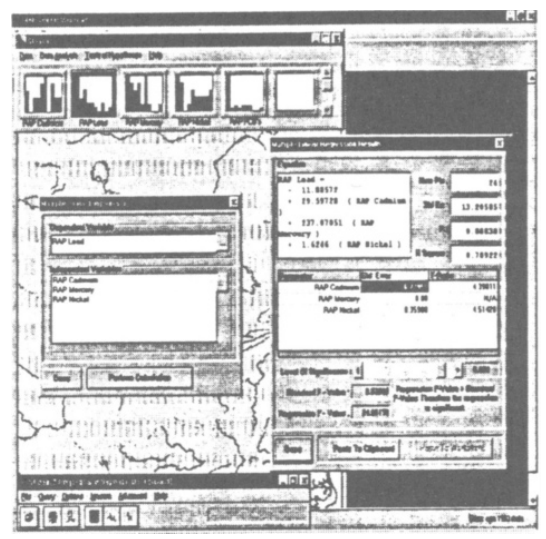

Figure 5 Statistical relationships among parameters may be determined using techniques such as multiple linear regression.

\section{Visualization}

The contouring module may be used to help to visualize the distribution of chemicals within the lakes. For example, we could return to the spatial query for Lake Ontario (Figure 6) and select the Background data and Tier I chemical lead (Lead (Pb)) using the SQL for years 1970-1996. Also shown in Figure 6 is a window that appears when a chemical is selected, in this case for lead ( $\operatorname{Lead}(\mathrm{Pb})$ ), which contains any associated metadata for that chemical. Here the analytical method used and QA/QC information is described. For spatial visualization of data, the data should have been collected at the same time. Consequently, the data is further filtered by selecting data that were collected during a single cruise made by a research ship on Lake Ontario. The data are then copied automatically to the system's spreadsheet and a site file is created for those data, which can be shown on a map of Lake Ontario, as shown in Figure 7. By selecting the contour module, the user has the option of performing either vertical or horizontal contours. If the vertical transect is selected, the user merely selects the sampling sites shown on the map by clicking on them with the mouse. After selecting from a number of options for colours and scale, and contouring method (e.g. inverse distance, Kriging (local or global)), the vertical cross section graph is created, as shown in Figure 7 for filtered lead. 


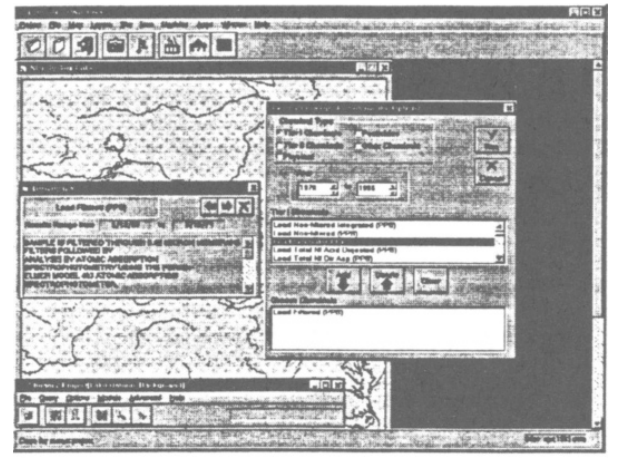

Figure 6 SQL query for filtered lead and associated metadata.

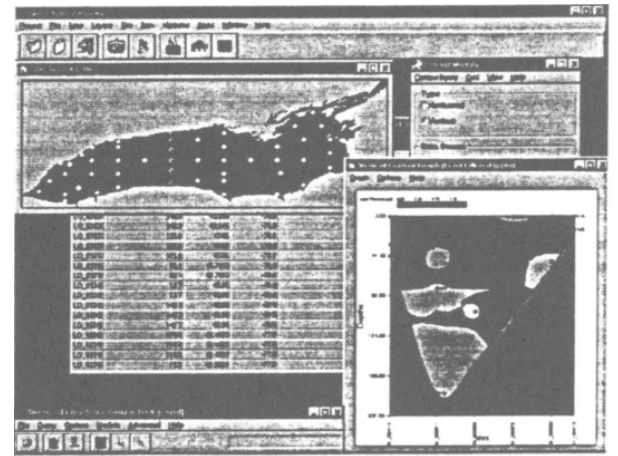

Figure 7 A vertical cross section contour graph for filtered lead data collected during Lake Ontario cruise C7122019.

\section{Modelling}

Models represent our knowledge of system processes. When calibrated and validated with observed data, they can be used to make projections, evaluate scenarios, etc. Models can be incorporated into the RAISON system by : (a) using the executable codes as given through appropriate interfaces for the model input and output, (b) emulating the model by a simplified version such as an input-output model, and (c) by converting the code to a programming language( e.g. Visual Basic) that can be run directly within the Windows environment. For example, as shown in Figure 8, a regional fugacity model (Mackay et al.) was incorporated into the system by converting it to Visual Basic.

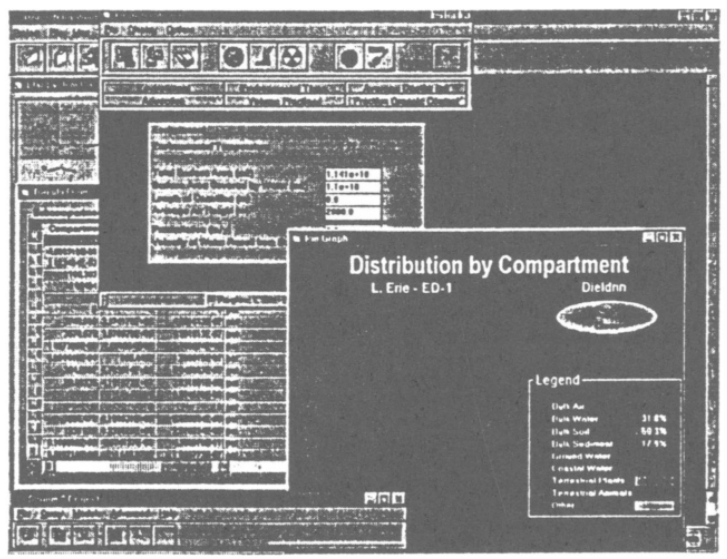

Figure 8 The fugacity model interface and visualization of model output showing the steady-state percent distribution of Dieldrin within the Lake Erie ecosystem. 
The input data for the model can be input directly from the database or can be input and edited through special windows created using Visual Basic, as shown in Figure 8. Also shown in Figure 8 is an example of one of the outputs for the chemical Dieldrin in Lake Erie. As a decision support tool, the model may also be linked with an optimization function such as a genetic algorithm or linear programming to find the minimum source reduction required to achieve a target level, subject to constraints such as best available technologies for emission reduction (Lam et al., 1995).

\section{FUTURE WORK}

The next phase of the project will involve the implementation of the trajectory models and the implementation of the rules for the expert system in dealing with the data and information classifications as well as the model applications

\section{ACKNOWLEDGEMENTS}

The authors are very grateful for assistance and contributions from the RAISON development team, especially, O. Resler, P. Fong, D. Kay, K. Brown, and F. Shiekh. This research was supported in part by the Environmental Protection Branch, Ontario Region, Environment Canada. Windows, Visual Basic and Access are trademark products of Microsoft Inc., USA MapInfo is a trademark product of the MapInfo Corporation and ArcInfo is a trademark product of the Environmental Systems Research Institute (ESRI) Inc.

\section{REFERENCES}

Booty, W.G., Wong, I.W.S., Lam, D.C.L., Kerby, J.P, and Kay, D.F. (1993) Application of an Expert System for Point Source Water Quality Modelling, in CSEIA 93-Computer Support for Environmental Impact Assessment (ed. G. Guariso and B. Page), Como, Italy. Elsevier, p.233-244.

Lam, D.C.L., Mayfield, C.I, Swayne, D.A. and Hopkins, K. (1994) A prototype information system for watershed management and planning. J. Biological Systems, 2(4):499-517.

Lam, D.C.L., Booty, W.G., Wong, I., Kay, D. and Kerby, J.P. (1995) RAISON decision support system: examples of applications. NWRI Contributing Report no. 95-151, Burlington, Ontario, Canada.

Mackay, D. , Patterson S. and Tam, D.D. (1991) Assessments of Chemical Fate in Canada: Continued Development of a Fugacity Model. A report prepared for Health and Welfare Canada.

\section{BIOGRAPHY}

Dr. Booty has been a research scientist with Environment Canada at the National Water Research Institute since 1988. His areas of research are geochemical modelling and decision support systems. He obtained his Ph.D. in Geology from McMaster University in 1983 and was a Research Assistant Professor at Clarkson University, Department of Environmental Engineering, from 1983 until 1988. 